\title{
A patient with MEN1-associated hyperparathyroidism, responsive to cinacalcet
}

Alberto Falchetti, Antonio Cilotti, Luca Vagelli, Laura Masi, Antonietta Amedei, Federica Cioppi, Francesco Tonelli and Maria Luisa Brandi

Nature Clinical Practice Endocrinology \& Metabolism (2008) 4: 351-357 [doi:10.1038/ncpendmet0816]

doi:10.1038/ncpendmet0888

CORRIGENDUM

In the June 2008 issue, the surname of the third author was published as "Vagelli". This should have appeared as "Vaggelli". 